\title{
EL MARKETING POR UNA MEJOR CALIDAD DE LOS SERVICIOS TURÍSTICOS EN CUBA
}

\author{
Seguí León Gladys Caridad'. \\ Rodríguez Miló Daymelis. \\ Universidad de Pinar del Rio (Cuba) \\ gladysc@fcsh.upr.edu.cu
}

Material original autorizado para su primera publicación en la revista académica REDMARKA. Revista Digital de Marketing Aplicado.

http://doi.org/10.17979/redma.2012.02.09.4753

Recibido: 12 Junio 2012

Aceptado 10 Diciembre 2012

\section{Resumen:}

Los principios y requisitos recogidos en el Marketing como ciencia que estudia el intercambio comercial entre los entes protagonistas del mismo, imponen a toda entidad de servicios la necesidad de controlar los niveles de satisfacción que tienen sus clientes con respecto a los servicios que oferta. El hotel Vueltabajo lleva a cabo un procedimiento para la recogida de esta información a través de la aplicación de una encuesta y la utilización de un libro de "Opinión del Cliente". Indagaciones realizadas permitieron corroborar las inquietudes existentes sobre la pertinencia y suficiencia del mismo. La presente investigación, la cual parte de un diagnóstico dirigido a puntualizar las limitaciones de la encuesta y del libro de

\footnotetext{
1 Gladys Caridad Seguí León. Profesora asistente del Departamento Comunicación Social y Periodismo, perteneciente a la Facultad de Ciencias Sociales y Humanísticas en la Universidad de Pinar del Río "Hermanos Saíz Montes de Oca", ha impartido durante 5 años las asignaturas de Marketing y marketing Social en la carrera Comunicación social, ha realizado numerosas investigaciones relacionadas con el marketing de los servicios y participado en varios eventos con dicha temática, en diciembre de 2011 participó y fue miembro del Comité Académico del "Primer Encuentro de Comunicadores Pinar del Río 2011".
} 
opinión, demuestra la necesidad de un procedimiento que permita recoger de manera exacta los estados de opinión de los clientes con respecto a los servicios que se brindan en la instalación. Es esta la causa fundamental que guía a la autora de la misma a proponer un procedimiento que permita medir con mayor precisión los niveles de satisfacción de los clientes del hotel, desde los aspectos del Marketing que tributen a estos niveles y que potencien el intercambio de información entre el cliente y la empresa, que en esencia son las sesiones en profundidad. En él se presentan las características principales para su cumplimiento en el marco de la instalación, se indican los pasos a seguir para realizar las sesiones, se aplica una nueva forma de examinar los resultados obtenidos y se diseñan los aspectos a tratar en las sesiones en profundidad. Acompaña a esto un modelo previamente aplicado a un grupo pilotaje para comprobar su alta confiabilidad.

Representa un valioso aporte debido a los resultados que sobre el conocimiento de los niveles de satisfacción del cliente se esperan con su aplicación, el cual propiciará poder encausar mejor todos los esfuerzos y usar los recursos en aras de un mayor bienestar para los clientes, razón de ser y fin de toda instalación de servicios.

Palabras Claves: servicio, instrumento, satisfacción.

\section{Abstract:}

MARKETING FOR A BETTER QUALITY OF TOURISM SERVICES IN CUBA

The principles and requirements picked up in the Marketing like science that studies the commercial exchange among the entities main characters of the same one, impose to all entity of services the necessity to control the levels of satisfaction that have their clients with regard to the services that it offers. The "Vueltabajo" hotel carries out a procedure for the collection of this information through the application of a survey and the use of a book of Clients' opinion. Carried out Inquiries allowed to corroborate the existent restlessness about the 
relevancy and sufficiency of the same one. The present investigation, which leaves of a diagnosis directed to remark the limitations of the survey and of the opinion book, it demonstrates the necessity of a procedure that allows to pick up in an exact way the states of the clients' opinion with regard to the services that are offered in the installation. It is the fundamental cause that guides the author of their work to propose a procedure that allows to measure with more precision of the clients' level of satisfaction in the hotel, from the aspects of the Marketing that pay at these levels and that potenciete the exchange of information between the client and the company that are the sessions in depth in essence. In it the main characteristics are presented for their execution in the mark of the installation, the steps are indicated to continue to carry out the sessions, a new form is applied of examining the obtained results and the aspects are designed to try in the sessions in depth. It accompanies this a model previously applied to a group pilotage to check their high dependability.

It represents a valuable contribution due to the results that it has more than enough the knowledge of the levels of the client's satisfaction they are expected with their application, which will propitiate to be able to prosecute all the efforts and to use better all the resources for the sake of a bigger well-being for the clients, reason of being and end of all installation of services.

Key Words: service, tool, satisfaction. 


\section{Introducción}

El Marketing significa algo más que vender los productos, o sea, es un proceso de doble sentido en el que juegan un papel primordial: el cliente, propiciador de información y la empresa como agente encargado de propiciar los bienes y servicios demandados.

La utilización exitosa del Marketing en Cuba tiene que transitar por un cambio en la cultura gerencial del país. Esto significa, partir de una estrategia clara y precisa como premisa básica para trabajar con alternativas de Marketing que permitan obtener mayores impactos con el mínimo de recursos, además servirá para insertar a Cuba con éxito en el entorno, así como para lograr pasos en la economía interna a fin de perfeccionar la producción y los servicios con una mejor orientación hacia la satisfacción de las necesidades y deseos de los clientes y la población en general.

La actividad turística en toda su extensión, no escapa de una filosofía de Marketing pues, su punto final es sin lugar a dudas el hombre y la satisfacción de sus necesidades de recreación, placer y esparcimiento, desenvolviéndose como un hecho de carácter social pero con implicaciones económicas muy significativas.

El Marketing turístico se convierte en el principal instrumento de que se dispone para estimular el funcionamiento del sistema turístico y para incrementar así, las corrientes turísticas hacia las regiones receptivas; de manera que de su eficacia depende, en gran medida, el éxito de los planes de desarrollo turístico emprendidos por Cuba.

Los esfuerzos del Estado Cubano relacionados con la actividad turística, específicamente en la esfera de los servicios orientados hacia la satisfacción de los clientes, han sido disímiles. Un ejemplo fehaciente es la provincia de Pinar del Río, que se caracteriza por poseer una exuberante naturaleza, montañas con cavernas de espectaculares formaciones secundarias, hermosos valles y llanuras, especies vegetales y animales de alto endemismo y belleza, fondos marinos envidiables para el buceo, así como 2 reservas de la biosfera: la Península de 
Guanahacabibes y La Sierra del Rosario; que hacen que sus incentivos turísticos fundamentales puedan resumirse como Naturaleza y que sus mercados aparezcan motivados por la recreación en espacios naturales y por una cultura e historia propia a fin de obtener una imagen particular y atractiva.

La Empresa Islazul Pinar del Río como estructura organizativa del Grupo Empresarial Hotelero Islazul S.A. fue creada el 30 de agosto de 1994, como resultado del acuerdo 14 de la Junta General de Accionistas de esta compañía para la administración, promoción y comercialización del producto turístico en el territorio pinareño.

Un análisis exploratorio realizado con clientes, directivos y trabajadores en general, permitió conocer sus criterios sobre el libro de Opinión del Cliente y la encuesta aplicada a los clientes como vías para medir el nivel de satisfacción de los mismos hacia los servicios que reciben y, aunque en el caso del libro las opiniones por lo general son favorables, en el caso de la encuesta emitieron criterios desfavorables en cuanto a: su confección y la precisión de su propósito, además de no tener en cuenta las opiniones dadas, con lo cual la misma no permite obtener una idea clara al respecto. De tales criterios emana también el hecho de que tanto los trabajadores como los directivos del hotel limitan el procedimiento empleado para medir los niveles de satisfacción solo a la aplicación de estos instrumentos.

Dicha indagación exploratoria condujo a las autoras de este trabajo a formular el siguiente problema ¿Cómo contribuir a medir con mayor precisión los niveles de satisfacción del cliente hacia los servicios turísticos que brinda el hotel Vueltabajo? Con el propósito de resolver el problema planteado se formuló como objetivo Proponer un procedimiento que permita medir con mayor precisión los niveles de satisfacción del cliente hacia los servicios turísticos del hotel Vueltabajo mediante la comunicación. Lo sobresaliente de los resultados radica en que a partir de los instrumentos aplicados a clientes, directivos y trabajadores en general, a fin de conocer la efectividad y calidad del procedimiento que utilizan en el hotel 
Vueltabajo para medir los niveles de satisfacción de sus clientes, se detectaron dificultades en el mismo. El análisis de los resultados permitió definir las principales fortalezas y debilidades de dicho procedimiento y evidenció la necesidad de sustituir la simple aplicación de una encuesta escrita y del libro de Opinión del Cliente, por un procedimiento que permita medir con mayor precisión los niveles de satisfacción del cliente hacia los servicios que recibe.

\section{Desarrollo:}

Cuba, destacada por una economía socialista y subdesarrollada se caracteriza por:

1. Ser un país del Tercer Mundo con las implicaciones socioculturales y de desarrollo económico que esto conlleva.

2. Poseer una economía interna de gran demanda y poca oferta con las consecuencias que esto tiene para la cultura de producción y la disciplina laboral.

3. Ser un país que ha producido un brusco reordenamiento en su comercio exterior a causa de la pérdida de cerca del $80 \%$ de sus relaciones comerciales.

4. Ser un país socialista con una economía planificada en la que la mayoría de las decisiones tienen un alto grado de centralización.

Es conveniente que se evalúen las implicaciones que para el Marketing tiene la situación económica de Cuba:

1. La escasez de recursos financieros del país y las limitaciones existentes para la importación de bienes de consumo, materias primas, materiales e insumos en general dificultan llevar a cabo la actividad económico productiva del país. 
Consecuentemente, una orientación al Marketing tendría implicaciones de racionalidad económica: no producir aquello que no va a satisfacer plenamente las necesidades y que implica gastos de recursos que no se recuperarían o que se lograrían solo parcialmente. Esto en términos de relaciones comerciales implica entonces la garantía de la realización de la producción y por tanto la segura obtención de fuentes de financiamiento.

En este sentido se debe tener en cuenta que en la economía coexisten dos formas de producción: una a partir de capital intensivo y en ocasiones hasta con tecnología de punta, propia de un país desarrollado y otra tradicional, primitiva, caracterizada por la manufactura y el comercio en pequeña escala, lo que obliga a dar tratamientos diferentes desde el punto de vista del Marketing a ambos subsistemas.

2. El propio hecho de que Cuba sea un país subdesarrollado es una razón más para que exista la necesidad de optimizar el uso de los recursos.

La virtual ausencia de un mercado interno no garantiza un laboratorio nacional, como sucede en otros países, para ensayar o estudiar el comportamiento de un producto previo a su lanzamiento al mercado internacional, ni presupone la existencia de un nivel de exigencia por parte de los consumidores que contribuya como factor de desarrollo de la competitividad nacional.

Por otra parte, la situación financiera del país obliga a acudir a la exportación no como resultado de un agotamiento de las posibilidades de crecimiento a partir del mercado interno de las empresas como resulta clásico, sino de manera obligada, lo cual tiene que ser visto más como un reto que como una limitación.

Entonces la práctica del Marketing en la economía nacional podría permitir entrenar a los empresarios cubanos para descubrir detrás de las modas del mercado necesidades e insatisfacciones humanas y aprender a no extrapolar hábitos y costumbres internas al comportamiento del mercado externo, con lo cual 
se aprende también a organizar estudios y sistemas de información de mercado útiles para aprovechar la información de mercado como retroalimentación para planificar la producción, los precios y la distribución de una manera más racional, no solo en el plano interno, sino en el externo para poder exportar con éxito.

Además, en el plano interno el Marketing brinda también algunas técnicas para reducir o reorientar la demanda de determinados productos y no limitarse al uso de los precios como única herramienta para este fin.

3. Sin dudas, el desbalance interno entre la oferta y la demanda atenta contra el concepto de producto, limitándolo a su función básica y por tanto a su aspecto tangible, lo que eleva el poder del productor e incide negativamente en la relación producción-consumo.

La posibilidad de ser competitivos tiene que asegurarse con productos y servicios cuya concepción, diseño y oferta en otros mercados asegure que estos estén dirigidos a la satisfacción de alguna necesidad y que reporten algún beneficio esperado por los clientes potenciales, que sean atractivos para su compra por otros mercados y por tanto, preferidos de alguna manera por sobre los que actualmente se ofertan.

Resulta imprescindible que se posea una ventaja competitiva sostenible en cada uno de los productos para poder lanzarlos al mercado internacional, ocupar un lugar y permanecer en él y para ello se debe, no solo conocer las normas y valores que prevalecen en las economías de los compradores, sino que hay que organizar el proceso de diseño, producción y comercialización de forma tal que su resultado sea compatible con las expectativas y exigencias de los posibles consumidores en esos países.

4. La falta de financiamiento, por pérdida de las relaciones tradicionales de comercio exterior, obliga a una competitividad con el resto del mundo que haga 
atractivos los productos que ofrece Cuba para su compra por los mercados externos.

5. Como país socialista el hecho de que los valores sociales y del hombre primen en las decisiones, no entra en contradicción con el planteamiento formal de base del Marketing en el capitalismo, donde la satisfacción de los deseos del cliente es la justificación económica y social de la existencia de la empresa y la base de la estabilidad económica está en determinar esos deseos y luego satisfacerlos.

Raúl Castro en la reunión anual sobre la marcha del Proceso de Perfeccionamiento (1995) reconoció al Marketing y utilizó el término mercadotecnia como una de las herramientas para estudiar posibles nuevos mercados y el logro de la eficacia y la competitividad necesarias para las producciones en calidad, precios y oportunidad.

\section{Resultados del diagnóstico}

\section{Resultados del análisis documental}

\section{Análisis del libro de Opinión del Cliente}

En el análisis del libro de Opinión del Cliente, se revisaron los últimos seis meses del año 2011 y los meses transcurridos del 2012. Las principales fortalezas y debilidades que se detectaron en la aplicación de este instrumento fueron:

\section{Fortalezas}

Se encuentra ubicado en un lugar estratégico al alcance de todos los clientes.

Libertad para su uso.

Carácter anónimo y voluntario del llenado del libro.

\section{Debilidades}


No comprende selección de la muestra.

No dirige al cliente hacia las cuestiones medulares dignas de destacar por lo que en ocasiones se pierde en mencionar cuestiones irrelevantes.

No hay concebido un espacio donde se ofrezcan explicaciones a los clientes sobre las inquietudes por ellos formuladas.

\subsection{Análisis de la encuesta}

En la encuesta que utiliza el hotel Vueltabajo para medir los niveles de satisfacción de sus clientes, también se detectaron fortalezas y debilidades en cuanto a su aplicación, debilidades que impiden cumplir a cabalidad los objetivos de la misma. Estas fueron:

\section{Fortalezas}

Lenguaje formal y de uso popular.

Contiene la solicitud de cooperación dirigida a los encuestados.

Posee un carácter libre, anónimo y voluntario.

Dualidad de idioma.

Frecuencia en su aplicación.

\section{Debilidades}

Constituye un documento escrito.

Su estructura no es la más adecuada.

Las escalas utilizadas son muy sencillas.

Predominio de preguntas cerradas.

Requiere de tiempo para ser contestada.

Escasa posibilidad de argumentación. 
No facilita la retroalimentación entre el encuestador y el encuestado.

No tributa ni a la comunicación oral ni a la extra-verbal.

No toma en cuenta en su aplicación las particularidades generales de los clientes como la edad, sexo, raza, nacionalidad, etc.

No recoge opiniones sobre el nivel de satisfacción en otros aspectos componentes del Marketing, como son la comunicación y los precios.

\subsection{Resultados de la entrevista a trabajadores del hotel Vueltabajo}

Para realizar la entrevista se utilizó una muestra de 20 trabajadores de un total de 26.

Las respuestas ofrecidas por todos los entrevistados a las primeras dos preguntas permitieron constatar que son trabajadores bien informados sobre las funciones, objetivos, fines y política de su centro de trabajo y que la dirección del hotel se preocupa por conocer y satisfacer las necesidades y deseos de sus clientes; pues ellos constituyen ente activo dentro de la vida laboral de la institución.

En la pregunta 1: los 20 entrevistados reconocen la utilización de un libro de Opinión del Cliente y la encuesta; así como la revisión de las respuestas dadas como el único procedimiento empleado por el hotel.

En la pregunta 2: no existe coincidencia significativa en cuanto al número de clientes a los que se les aplican los instrumentos, sin embargo todos reconocen que el libro de "Opinión del Cliente" se aplica diariamente pues se encuentra ubicado en un lugar de fácil acceso para todos y en cuanto a la encuesta 14 (70\%) entrevistados afirman que se aplica semanalmente, tres (15\%) que se aplica quincenalmente y tres (15\%) diario, coincidiendo todos en que ambos instrumentos son aplicados bajo un ambiente afable y de total flexibilidad.

En la pregunta 3: el 100\% de los entrevistados afirman que el objetivo de la aplicación de este procedimiento va dirigido a conocer las opiniones que tienen los 
clientes sobre la calidad de los servicios que presta la instalación, para así poder enfocar mejor sus esfuerzos y recursos hacia la satisfacción de los mismos.

En la pregunta 4: todos los entrevistados consideran que el libro de "Opinión del Cliente" cumple con el objetivo para el cual fue creado, sin embargo 12 (60\%) de los entrevistados expresan sus reservas en cuanto al cumplimiento del objetivo declarado en la encuesta y 8 (40\%) argumentan que la misma refleja ciertas deficiencias que limitan el conocimiento cabal de los niveles de satisfacción del cliente.

En la pregunta 5: de los 20 entrevistados, cuatro (20\%) expresan que los instrumentos no presentan problemas y/o limitaciones que afecten el procedimiento empleado para medir los niveles de satisfacción de los clientes, mientras que los restantes $16(80 \%)$ mencionan como problemas y limitaciones las siguientes:

\section{Libro de Opinión del Cliente}

No guía al cliente hacia qué aspectos referirse.

\section{Encuesta}

Induce a respuestas breves, rígidas y con bajo nivel de argumentación.

Algunas preguntas no dejan claras sus intenciones.

Las dimensiones relacionadas no contemplan todos los indicadores que les corresponden.

Este tipo de instrumento no permite el intercambio de criterios entre cliente y trabajadores.

El análisis de las respuestas es muy pobre lo cual le resta efectividad al instrumento. 
En la pregunta 6: los 20 trabajadores entrevistados afirman que la ejecución de este procedimiento es la primera prioridad en el hotel, pues a partir de él se tiene una idea más clara de la calidad con que se prestan los servicios y de la satisfacción de sus clientes para con ellos.

En la pregunta 7: ninguno de los 20 entrevistados posee criterios de confiabilidad y validez de los instrumentos.

\subsection{Resultados de la entrevista a directivos del hotel Vueltabajo}

Fueron entrevistados dos directivos. Ambos mostraron con sus respuestas a las tres primeras preguntas poseer un conocimiento pleno sobre la existencia, aplicación y pertinencia de los instrumentos utilizados en el hotel para conocer los niveles de satisfacción de los clientes del mismo.

En la pregunta 4: los directivos mencionan solo el libro de "Opinión del Cliente" y la encuesta; así como el control de las respuestas dadas como único procedimiento para medir los niveles de satisfacción del cliente.

En la pregunta 5: ambos directivos coincidieron en que la encuesta se aplica semanalmente y que el libro de Opinión del Cliente se encuentra abierto permanentemente al alcance de los clientes enfatizando en que se emplean en un ambiente tranquilo, agradable y flexible. Nada dicen del análisis posterior de los resultados.

Del mismo modo en sus respuestas a la pregunta 6 afirman que son aplicados los instrumentos a todo tipo de clientes.

En la pregunta 7: ambos directivos afirman que el objetivo fundamental del procedimiento es conocer los gustos, preferencias, deseos y niveles de satisfacción de los clientes con respecto a los servicios que brinda la instalación; argumentando convenientemente. 
En la pregunta 8: consideran que los instrumentos utilizados si recopilan de manera eficiente las opiniones de los clientes, aunque coinciden en afirmar que a la encuesta podrían introducírseles algunos indicadores que hagan alusión a aspectos no recogidos en la estructura actual.

En la pregunta 9: respecto a la estructura y al estilo del libro de "Opinión del Cliente", los dos plantean que a su consideración está acorde con las necesidades de la instalación. Sin embargo con respecto a la encuesta, uno (50\%) de ellos expresa que su estructura no es la más adecuada pues no tiene concentrada en una sola parte las preguntas a responder por los clientes no siendo compartido ese criterio por el otro directivo y en cuanto al estilo argumentan que posee un lenguaje asequible para todos y que en ella solo se recogen criterios sobre los servicios que brinda el hotel.

En la pregunta 10: los dos entrevistados destacan como fortaleza la existencia de ambos instrumentos y como debilidades, en cuanto al libro de "Opinión del Cliente" ninguno hace señalamiento. Sin embargo en cuanto a la encuesta, uno (50\%) de los dos considera que como instrumento no permite recoger las opiniones del modo más veraz posible, que para su aplicación no se toman en cuenta los datos que permiten identificar las particularidades de cada cliente, que no posibilita la argumentación de las respuestas y que las preguntas son sumamente estrictas, mientras que el otro (50\%) afirma que si cumple con sus objetivos. No realizan más inferencias sobre el procedimiento en su conjunto.

En la pregunta 11: la dirección del hotel reconoce que la aplicación de este procedimiento es la prioridad número uno de la instalación, pues argumenta que a partir de los resultados obtenidos de la utilización de los instrumentos se pueden proyectar acciones dirigidas a erradicar o al menos minimizar las dificultades detectadas en la prestación de los servicios con el objetivo de que estos sean superiores y que se ofrezcan con la calidad requerida. 
En la pregunta 12: ambos directivos coinciden con que en el hotel no existe ningún especialista o departamento especializado que se ocupe de aplicar los instrumentos y analizar los resultados.

En la pregunta 13: ninguno de los directivos ofrece respuesta a esta pregunta.

En la pregunta 14: ambos afirman que el procedimiento seguido por el hotel para medir los niveles de satisfacción de los clientes consiste en la aplicación de dos instrumentos (libro de "Opinión del Cliente" y la encuesta) y una posterior revisión de las respuestas dadas con el fin de darle solución a las inconformidades teniendo en cuenta las posibilidades del hotel.

\subsection{Resultados de la encuesta a clientes del hotel Vueltabajo}

De los 33 clientes aproximadamente que entraron diariamente a la instalación en el mes de enero fueron encuestados 110 clientes, los cuales en las primeras cuatro preguntas demostraron tener dominio de los servicios que brinda el hotel, expresaron cuáles de ellos en su opinión fueron los mejores brindados y las motivaciones que los condujeron a consumir dichos servicios.

En la pregunta 5: los 110 clientes reconocen la encuesta y el libro de Opinión del Cliente como los instrumentos que aplica el hotel Vueltabajo para medir los niveles de satisfacción. No citan otro elemento del procedimiento de medición.

En la pregunta 6: la totalidad de los que reconocen la encuesta afirman que la misma es aplicada antes de abandonar el hotel, 90 (81,8\%) mencionan que el libro de Opinión del Cliente se aplica diario porque se encuentra ubicado en un lugar accesible a todos y 20 (18,2\%) se abstienen de dar criterios. Los 110 encuestados destacan que el procedimiento se aplica en el lugar y momento apropiado, con un trato agradable y con total facultad para rechazar el llenado de la encuesta si se desea.

En la pregunta 7: De los 110 encuestados, 100 (90,9\%) responden que el libro de Opinión del Cliente les permite expresar sus criterios de forma completa, mientras

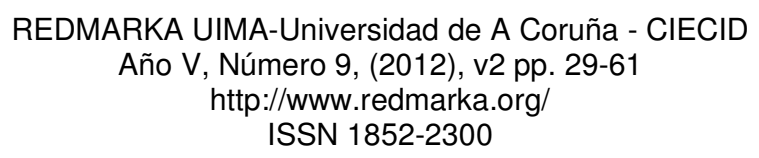


que los restantes $10(9,1 \%)$ abordan que no se sienten seguros a la hora de dar un criterio pues no tienen parámetros que los guíen. De los 110 que reconocen la encuesta, 90 (81,8\%) responden que la misma no tiene esa posibilidad, 15 $(13,6 \%)$ responden afirmativamente y cinco $(4,5 \%)$ se abstienen.

En la pregunta 8: de los que reconocieron la encuesta 27 (24,5\%) respondieron que los parámetros que se exponen en la misma son suficientes, 73 (66,4\%) afirman que no y $10(9,1 \%)$ no emiten opinión. En cuanto al libro de Opinión del Cliente no hay respuestas en este sentido.

En la pregunta 9: de los 110 encuestados, 30 (27,3\%) no sugieren nada con respecto a la encuesta, $73(66,4 \%)$ proponen ideas como: otorgar mayor amplitud a las preguntas, ofrecer mayor posibilidad de explicación, que sean dialogadas las respuestas, que las preguntas ofrezcan más nivel de información para que resulten más claras y ganen en precisión y siete $(6,4 \%)$ se abstiene. En cuanto al libro de Opinión del Cliente no ofrecen criterios.

En la pregunta 10: los 110 encuestados que reconocen el libro de Opinión del Cliente coinciden en que este instrumento tiene gran importancia pues les permite expresar libremente sus criterios sobre los servicios recibidos, igualmente ocurre con la encuesta.

En la pregunta 11: en cuanto al libro de Opinión del Cliente de los 110 que lo reconocen, 60 (54,5\%) expresan que este instrumento no ofrece posibilidad de asesoría que les permita a ellos terminar de conformar y precisar sus opiniones, $30(27,3 \%)$ plantean que sería conveniente que este libro presentara los aspectos sobre los cuales el hotel pretende recoger las opiniones y se abstienen a emitir un criterio 20 (18,2\%). Por otra parte, de los 110 que reconocen la encuesta, 31 $(28,2 \%)$ de ellos no reconocen problema alguno en la misma; pero entre los 79 $(71,8 \%)$ restantes hacen alusión a una serie de problemas y/o inconvenientes presentes en este instrumento. No hacen referencia a nada más, mostrando que esto es lo único que conocen como procedimiento. 
En la pregunta 12: de los 110 clientes que reconocen la encuesta y el libro de "Opinión del Cliente" la totalidad afirmó que la calidad del último instrumento es de buena, mientras que con relación a la encuesta 30 (27,3\%) la califican de buena, $65(59,9 \%)$ de regular y $15(13,6 \%)$ se abstienen.

En la pregunta 13: ninguno de los encuestados dijo conocer otra forma.

\section{Análisis de los resultados del diagnóstico}

Después de realizado el diagnóstico situacional se procede al análisis de los resultados para lo cual se comienza por efectuar una triangulación de los resultados obtenidos de los instrumentos aplicados a fin de conocer el nivel de coincidencia de las respuestas dadas a la variable y dimensiones objeto de estudio.

Triangulación de los tres instrumentos aplicados a los diferentes protagonistas del sistema (clientes, trabajadores y directivos)

\section{Existencia de los instrumentos}

Todos reconocen el libro de Opinión del Cliente para un 100\% de coincidencia.

La encuesta la reconocen los 20 trabajadores, los dos directivos y 110 clientes para un $100 \%$ de coincidencia.

\section{Declaración del objetivo}

Los 20 trabajadores y los dos directivos consideran los objetivos bien definidos para un $100 \%$ de coincidencia.

\section{Recopilación de las opiniones}

$14(70 \%)$ trabajadores, los 110 (100\%) clientes y dos directivos (100\%) coinciden en que en la encuesta se recogen opiniones sinceras. 
90 clientes $(81,8 \%)$ afirman que la encuesta no les permite ofrecer su respuesta completa al indicar respuestas puntuales y solo por escalas.

14 trabajadores (70\%) y 10 (9\%) clientes coinciden en que el libro de "Opinión de Cliente" no permite al cliente enfocar sus opiniones.

\section{Problemas y/o limitaciones del procedimiento}

16 trabajadores (80\%) le reconocen problemas o limitaciones a la encuesta coincidiendo con la opinión de un directivo (50\%) y 79 clientes $(71,8 \%)$.

Cuatro trabajadores (20\%), 31 clientes $(28,2 \%)$ y un directivo $(50 \%)$ coinciden en que la encuesta no presenta problemas o limitaciones.

En cuanto al libro de "Opinión del Cliente" cuatro trabajadores (20\%) y dos directivos (100\%) coinciden en que no presenta problemas o limitaciones.

16 trabajadores (80\%) y 90 clientes $(81,9 \%)$ coinciden en que el libro si presenta limitaciones.

\section{Prioridad del procedimiento}

Tanto los trabajadores entrevistados, como los dos directivos reconocen que la aplicación de este procedimiento constituye una prioridad para un $100 \%$ de coincidencia.

\section{Importancia del procedimiento}

17 trabajadores (85\%) y 110 clientes (110\%) ofrecen criterios que reflejan de algún modo la importancia del procedimiento empleado para recoger las opiniones expresadas sobre la calidad de los servicios.

\section{Confiabilidad y validez del procedimiento}


Ni los dirigentes ni los trabajadores conocen criterio de confiabilidad y validez de los instrumentos aplicados para el $100 \%$ de coincidencia.

\section{Esencia del procedimiento}

Tanto los trabajadores como los directivos reconocen como procedimiento para medir los niveles de satisfacción del cliente la simple aplicación de los dos instrumentos mencionados y el posterior conteo de las respuestas según categoría asignada, para el $100 \%$ de coincidencia. Los clientes lo limitan a la sola aplicación de los instrumentos.

El análisis documental y la triangulación efectuada a los instrumentos aplicados permitieron a las autoras focalizar las principales fortalezas y debilidades del procedimiento para medir los niveles de satisfacción del cliente que se lleva a cabo en el hotel Vueltabajo.

\section{Fortalezas}

\section{Libro de Opinión del Cliente”}

Existencia de un libro para recoger las opiniones de los clientes sobre la calidad de los servicios.

Acertada ubicación del libro de Opinión del Cliente y oportuna promoción del mismo.

Carácter libre, anónimo y voluntario del llenado del libro.

Permite que los clientes expresen sus opiniones de modo sincero.

Objetivos bien definidos.

Su aplicación es de primera prioridad.

Es considerado de importancia porque posibilita expresar libremente criterios sobre los servicios. 


\section{Encuesta}

Existencia y aplicación de la encuesta con similar propósito al del libro de Opinión del Cliente.

$\square$ El lenguaje utilizado es formal y de uso popular. Posibilita encuestar un mayor número de clientes en un menor tiempo relativo.

Cumple con el requisito de solicitud de cooperación dirigido a los encuestados.

Frecuencia de aplicación.

Carácter libre, anónimo y voluntario del llenado de la encuesta.

Objetivos bien definidos.

Posibilita la recolección de opiniones sinceras.

Su aplicación es una prioridad tanto para trabajadores como directivos del hotel.

Se le atribuye importancia por la libertad que ofrece para expresar los criterios.

\section{Debilidades}

\section{Generales:}

Existe en todos los trabajadores y directivos del hotel una noción simplista del procedimiento para medir los niveles de satisfacción de los clientes, limitándolo a la simple aplicación de los instrumentos citados y una somera revisión de las respuestas dadas, lo que le confiere muy poca profundidad al procedimiento seguido.

Los instrumentos se aplican sin un conocimiento previo de los niveles de confiabilidad y validez de los mismos.

Aún cuando los instrumentos tributan a los objetivos propuestos en ellos, el procedimiento seguido para medir los niveles de satisfacción, al quedar limitado como ya se explicó, no es consecuente con estos fines. 
Ninguno de los dos instrumentos potencia el intercambio de opiniones y criterios entre los clientes y los trabajadores.

\section{Libro de Opinión del Cliente}

$\square$ El libro no brinda posibilidades de asesoría al cliente que le permita dirigir sus opiniones hacia las cuestiones más importantes para el hotel.

No comprende selección de la muestra.

\section{Encuesta}

Constituye un documento escrito.

Le es aplicada a una ínfima porción de los clientes.

Su estructura no es la más adecuada.

Las escalas utilizadas son muy sencillas.

Predominio de preguntas cerradas.

No toma en cuenta para su aplicación las particularidades generales de los clientes como la edad, sexo, raza, nacionalidad, etc.

Las respuestas recogidas no pueden ser aclaradas ni corregidas para evitar diversidad de interpretaciones.

No posibilita la retroalimentación.

No tributa ni a la comunicación oral ni a la extra-verbal.

No ofrece facilidad de argumentación de las respuestas.

No recoge opiniones sobre el nivel de satisfacción en otros aspectos componentes del Marketing, como son la comunicación y los precios.

Requiere de tiempo para ser contestada. 
El análisis de los resultados del diagnóstico situacional del procedimiento para medir los niveles de satisfacción del cliente evidencia la necesidad de sustituir la simple aplicación de estos instrumentos por un procedimiento que permita medir con mayor precisión los niveles de satisfacción del cliente en el hotel Vueltabajo en los aspectos del Marketing que tributen directamente a dichos niveles y a la comunicación.

\section{Necesidad de un instrumento más confiable.}

La encuesta es una interacción escrita que cumple con la condición de ser tangible y verificable, además puede almacenarse por tiempo indefinido y suele estar mejor pensada y ser más lógica y clara.

Sin embargo los resultados obtenidos del diagnóstico realizado en el hotel Vueltabajo a directivos, trabajadores y clientes en general acerca de la efectividad del procedimiento que allí se aplica, demuestran que el mismo, limitado únicamente a la aplicación de la encuesta y del libro de Opinión del Cliente, no recoge de forma precisa y exacta los niveles de satisfacción de los clientes con respecto a los servicios turísticos que este brinda. Tal situación ha inducido a las autoras de este trabajo a la elaboración de un procedimiento más eficaz en el cumplimiento de los fines descritos con anterioridad. En opinión de las misma, por sus potencialidades comunicativas, son las sesiones en profundidad el instrumento idóneo.

Dadas las características de los entrevistados en este caso, se propone que la sesión o sesiones de grupos se realicen con carácter informal por parte de un especialista que dialogue con pequeños grupos en los propios lugares de esparcimiento de modo breve, ameno, espontáneo, concreto y con énfasis en lo afectivo fomentando la comunicación directa grupal y recogiendo el contenido de la conversación en grabaciones de las cuales se extraigan regularidades 
posteriormente al realizar el análisis de contenido. El procedimiento a seguir es el siguiente.

\section{Pasos para realizar las sesiones de grupo}

Se define el tipo de personas que habrán de participar en la sesión o sesiones.

Se detectan personas del tipo elegido.

Se invita a estas personas a la sesión o sesiones.

Se organizan la sesión o sesiones. Cada sesión debe efectuarse en un lugar confortable, silencioso y aislado. Los sujetos deben sentirse cómodos y relajados. Asimismo, es indispensable planear cuidadosamente lo que se va a tratar en la sesión o sesiones (desarrollar una agenda) y asegurar los detalles (aún las cuestiones más sencillas como servir café y refrescos).

Se lleva a cabo cada sesión. El conductor debe ser una persona entrenada en el manejo o conducción de grupos y debe crear rapport en el grupo (clima de confianza). Asimismo, debe ser un individuo que no sea percibido como "distante" por los participantes de la sesión y debe propiciar la participación de todos. La paciencia es una característica que deberá tener.

Durante la sesión se pueden pedir opiniones, hacer preguntas, discutir casos, intercambiar puntos de vista, valorar diversos aspectos. Es conveniente que cada sesión se grabe en cinta y después realizar análisis de contenido y observación. El conductor debe tener muy en claro la información o datos que habrán de recolectarse y evitar desviaciones del objetivo planteado.

Elaborar el reporte de sesión. El cual incluye principalmente datos sobre los participantes (edad, sexo, nivel educativo y todo aquello que sea relevante para el estudio), fecha y duración de la sesión, información completa del desarrollo de la sesión, actitud y comportamiento de los participantes hacia el conductor y la 
sesión en sí, resultados de la sesión y observaciones del conductor, así como una bitácora de la sesión.

Llevar a cabo la codificación y análisis correspondiente. Las preguntas deben codificarse con símbolos o números porque de lo contrario no puede efectuarse ningún análisis o sólo se puede contar el número de respuestas en cada categoría. Los datos deben resumirse, codificarse y prepararse para el análisis.

De acuerdo con los objetivos que se persiguen se considera oportuno utilizar la entrevista semi-estandarizada.

Una vez realizadas las sesiones en profundidad se recomienda el análisis de contenido.

\section{Propuesta}

\begin{tabular}{|c|c|c|c|c|}
\hline Categorías & $\begin{array}{l}\text { Definición } \\
\text { Operacional }\end{array}$ & Dimensiones & Indicadores & Ítems \\
\hline \multirow[t]{5}{*}{ Publicidad } & \multirow[t]{5}{*}{$\begin{array}{l}\text { Actividad de } \\
\text { comunicación } \\
\text { comercial } \\
\text { remunerada. }\end{array}$} & \multirow[t]{2}{*}{ Informativa } & Clara & \multirow{2}{*}{$\begin{array}{l}\text { ¿Cómo llegó } \\
\text { a su } \\
\text { conocimiento } \\
\text { nuestra oferta } \\
\text { hotelera? }\end{array}$} \\
\hline & & & Precisa & \\
\hline & & \multirow[t]{3}{*}{ Afectiva } & Confianza & \multirow{3}{*}{$\begin{array}{l}\text { Describa la } \\
\text { motivación } \\
\text { que lo } \\
\text { condujo a } \\
\text { escoger esta } \\
\text { oferta. }\end{array}$} \\
\hline & & & & \\
\hline & & & Atracción & \\
\hline \multirow{2}{*}{$\begin{array}{l}\text { Calidad de } \\
\text { los servicios }\end{array}$} & & \multirow[t]{2}{*}{ Oferta } & Calidad & \\
\hline & & & Presencia & \\
\hline
\end{tabular}




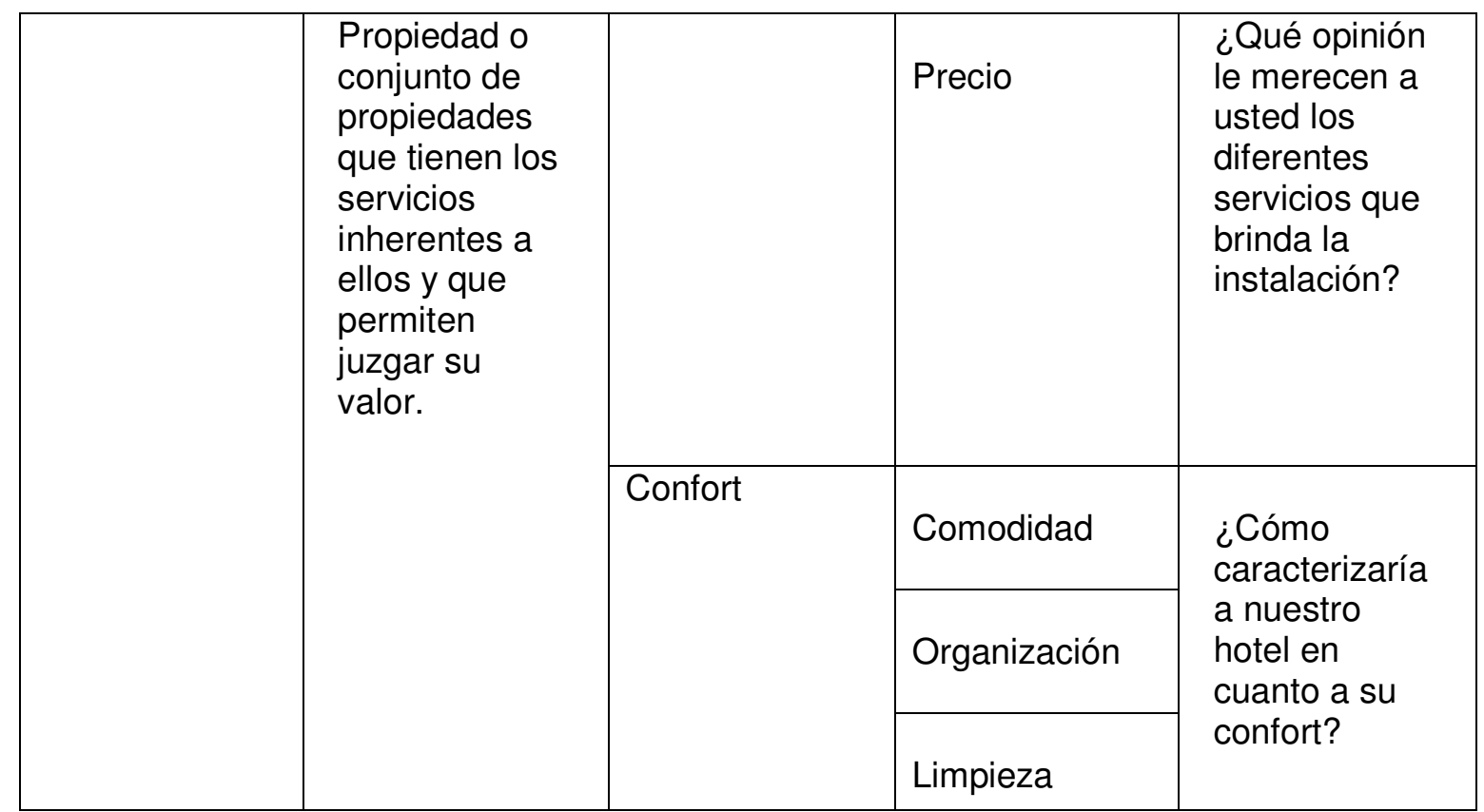

\section{Ventajas de la propuesta}

Promueve la comunicación directa grupal.

Cumple con el proceso de comunicación (emisor, codificación, mensaje, canal, decodificación, receptor y retroalimentación).

Favorece la rapidez en la obtención de la información y la retroalimentación.

Beneficia la comunicación oral y la comunicación no verbal.

Se pueden pedir opiniones, hacer preguntas, discutir casos, intercambiar puntos de vista y valorar diversos aspectos.

Las respuestas pueden ser aclaradas y corregidas para evitar diversidad de interpretaciones.

$\square$ Garantiza la calidad, intercambio y veracidad de la información.

$\square$ Ofrece posibilidades de argumentación de las respuestas.

Recoge opiniones sobre los niveles de satisfacción de los clientes en otros aspectos componentes del Marketing (4P's) como la comunicación y los precios y sobre la mezcla de este en los servicios. 
El procedimiento que se propone tiene una validez probada a partir de las opiniones recogidas entre el personal acreditado en el mundo del turismo sobre los ítems que lo conforman. Del mismo se comprobó su alta confiabilidad mediante la medición de su estabilidad a través de la aplicación de un test-retest en un pilotaje efectuado con un grupo de clientes del hotel consistente en aplicarles el procedimiento en dos momentos espaciados en un intervalo de tiempo de 15 días, constatándose una correlación de un 90\% entre las respuestas dadas.

\section{Conclusiones}

1. Los resultados del diagnóstico realizado a la situación actual del objeto permitieron concluir que:

La satisfacción del cliente como parte fundamental y esencial del hotel Vueltabajo, constituye la principal preocupación de este para el logro de sus objetivos.

El procedimiento empleado por el hotel Vueltabajo no mide de forma precisa los niveles de satisfacción de los clientes con respecto a los servicios que este brinda.

2. El análisis de los resultados permite fundamentar la necesidad de sustituir el procedimiento utilizado por el hotel Vueltabajo por otro procedimiento que permita medir con mayor precisión los niveles de satisfacción del cliente hacia los servicios turísticos que reciben, en los aspectos del Marketing que tributen directamente a dichos niveles y a la comunicación.

3. Se espera que la aplicación del procedimiento propuesto permita a la instalación estar más informada sobre lo que el cliente realmente prefiere, desea y necesita para sentirse satisfecho, enfrentar de forma más competente cualquier actividad relacionada con la satisfacción de este, tener una medida exacta del comportamiento y calidad de los servicios y por consiguiente que el cliente se sienta identificado con los servicios que se ofertan. 


\section{Bibliografía}

Ambrosio, V. (2000). Plan de marketing paso a paso. Una guía práctica para lanzar con éxito los productos. Colombia: Pretince Hall.

Alonso, M. (1998). Selección de textos sobre Metodología de la investigación cuantitativa. Cuba: La Habana.

Alonso, M., Saladrigas, H. (2000). Metodología de la Investigación Social Facultad de Comunicación. . Cuba: La Habana.

Allen, D. (1994). Desarrollo con éxito de nuevos productos. Nueva Cork: McGrawHill.

Alfaro, T. (1994). El Marketing como arma competitiva. México: McGraw-Hill Interamericana de México, S. A.

Arellano, Cueva, R. (2002). Comportamiento Del Consumidor: México: McGrawHill.

Barquero, J.D. (2000). Relaciones públicas financieras. España: Barcelona, Editorial Gestión. (2000). Manual de Relaciones Públicas empresariales e institucionales. España: Barcelona, Editorial Gestión.

Barba, E. (1993). La excelencia en el proceso de desarrollo de nuevos productos. España: Barcelona, Editorial: EADA Gestión.

Barón, L. (1982). Fundamentos del Marketing. España: Madrid. Editorial ICAI.

Benavides, J. (2001). Problemas conceptuales y metodológicos en el ámbito del Director de Comunicación en: Dirección de Comunicación Empresarial e Institucional, España: Barcelona. Editorial Gestión 2000, S.A.

Bell, Martin, L. (1982). Mercadotecnia, conceptos y estrategia. EUA: Washington University.

Blackwell, D. y otros (2002). Comportamiento del Consumidor. México: Thomson, 9a Edición. 


\section{Anexos}

\section{Entrevista aplicada a trabajadores del hotel Vueltabajo Objetivo}

Recopilar los estados de opinión de los trabajadores del hotel Vueltabajo con respecto al procedimiento que utiliza dicho hotel para medir los niveles de satisfacción de sus clientes hacia los servicios recibidos.

La finalidad de esta entrevista es la de poder intercambiar con usted acerca del procedimiento que lleva a cabo el hotel para medir los niveles de satisfacción de sus clientes. Sus opiniones serán valoradas, respetadas y de gran importancia para la investigación.

\section{Cuestionario}

1. ¿A través de qué procedimiento el hotel logra recoger el estado de opinión de sus clientes con respecto a los servicios que se brindan en el mismo?

2. ¿A cuántos clientes se les aplican los instrumentos para efectuar el procedimiento de medición y con qué frecuencia y rigor?

3. ¿Conoce usted con qué objetivo(s) la institución ejecuta dicho procedimiento?

4. ¿Cree usted que el objetivo(s) se cumplen a cabalidad? ¿Por qué?

5. ¿Qué problemas y/o limitaciones se presentan en el procedimiento para medir los niveles de satisfacción del cliente?

6. ¿Qué nivel de prioridad tiene la ejecución de este procedimiento para la dirección del hotel y trabajadores en general?

7. ¿Qué criterio se sigue para determinar la confiabilidad y validez de los instrumentos aplicados?

\section{Entrevista aplicada a directivos del hotel Vueltabajo \\ Objetivo}

Recoger las opiniones expuestas por los directivos del hotel Vueltabajo acerca del procedimiento que utiliza dicho hotel para medir los niveles de satisfacción de sus clientes con respecto a los servicios brindados. 
La finalidad de esta entrevista es la de poder recopilar el estado de opinión de usted, como factor fundamental dentro de la entidad, acerca del procedimiento que lleva a cabo el hotel para medir los niveles de satisfacción de sus clientes. Sus opiniones serán valoradas, respetadas y de gran importancia para la investigación. Cuestionario

1. ¿Qué entiende usted por satisfacción del cliente?

2. ¿Cuáles son los clientes asiduos del hotel y cómo es su relación con ellos?

3. ¿Cuáles son los canales que utiliza el hotel para comunicarse con sus clientes y conocer sus necesidades, deseos y preferencias con respectos a los servicios que brinda el mismo?

4. ¿Qué procedimiento sigue el hotel Vueltabajo para medir los niveles de satisfacción de sus clientes?

5. ¿Con qué frecuencia y rigor se realiza este procedimiento?

6. ¿Dicho procedimiento se realiza con todo tipo de clientes o se centra principalmente en una pequeña porción del total?

7. ¿Cuáles son los objetivos que se persigue con este procedimiento?

8. ¿Cree usted que los instrumentos utilizados recopilen de manera eficiente las opiniones que arrojan los clientes? ¿Por qué?

9. ¿Cree usted que la estructura y el estilo de los instrumentos sean los adecuados?

10. ¿Cuáles son, en su opinión, las fortalezas y las debilidades de los instrumentos?

11. ¿Qué nivel de prioridad tiene la aplicación de este procedimiento para la dirección del hotel y trabajadores en general?

12. ¿Existe un especialista, departamento o grupo de comunicación que se ocupe de aplicar y analizar los resultados arrojados en dichos instrumentos?

13. ¿Qué criterio se sigue para evaluar la confiabilidad y validez de los instrumentos utilizados en el procedimiento seguido para medir los niveles de satisfacción del cliente? 
14. ¿En qué consiste, en general el procedimiento seguido por el hotel para medir los niveles de satisfacción del cliente?

\section{Encuesta aplicada a clientes del hotel Vueltabajo Objetivo}

Recopilar los criterios que, sobre el procedimiento que utiliza el hotel Vueltabajo para medir los niveles de satisfacción, tienen los clientes que visitan dicha instalación.

Se requiere de su colaboración con el fin de indagar sobre el procedimiento que utiliza el hotel Vueltabajo para medir los niveles de satisfacción de sus clientes. Su criterio resultará de gran importancia para la investigación.

Instrucciones para el encuestado:

Escriba con letra clara.

Lea detenidamente las preguntas y las indicaciones que aparecen entre paréntesis (). Responda en correspondencia con su más sincera opinión.

Cuestionario

1. ¿Conoce usted los servicios que brinda el hotel "Vueltabajo"?

Sí__ No

En caso de conocerlos, menciónelos:

2. ¿Cuál de los servicios ha consumido?

3. ¿Cuál 0 cuáles fueron los servicios mejores brindados?

4. ¿Qué lo motivó a utilizar los servicios de este hotel?

(Marque tres respuestas como máximo) Relación calidad-precio. Prestigio del hotel. Recomendaciones de amigos. Calidad del servicio.

Desconocimiento de otros hoteles con similares servicios. Ubicación de la instalación (cercanía a lugares de su interés). Visitas anteriores / Conocimiento de la instalación. 
Tranquilidad / Seguridad.

Negocios/Trabajo.

Otros motivos:

5. ¿Qué procedimiento utiliza el hotel para conocer su satisfacción a partir de los servicios que este brinda?

6. ¿Con qué frecuencia y rigor les son aplicados los instrumentos concebidos en el procedimiento para medir los niveles de satisfacción?

Frecuencia Rigor

Diario _ Severo

Mensual__Afable

Trimestral__ Ninguno

Anual

7. ¿Cree usted que estos instrumentos permiten exponer de forma completa sus criterios?

$\mathrm{Si}$

8. ¿Son suficientes los parámetros que se exponen en dichos instrumentos?

$\mathrm{Si} \_$No

9. ¿Según su opinión, que le sumaría o le restaría a este procedimiento para que sea más eficaz a la hora de recoger las opiniones?

10. ¿Qué importancia tiene para usted la ejecución de dicho procedimiento?

11. ¿Qué problemas y/o inconvenientes les ve a este procedimiento?

12. ¿Cómo calificaría usted la calidad del mismo?

$B$

$\mathrm{R}$

M

13. ¿Conoce de otra forma empleada por el hotel para conocer sus criterios sobre la calidad de los servicios prestados?

Por su cooperación muchas gracias. 


\section{Interview that was applied to the clients of "Vueltabajo" hotel Objective}

To compile the criterion about the procedure that are used in "Vueltabajo" hotel for measuring the levels of satisfaction in clients that have visited this installation.

It is very important your contribution to carry out the purpose of this investigation about the procedure used in "Vueltabajo" hotel to measure the clients' levels of satisfaction by the service received, then your criterion is very important.

Instructions for the one interviewed:

Write with clear letter.

Read the questions and the indications that appear between parenthesis (). Respond in correspondence with your most sincere opinion.

Questionnaire:

1. Do you know the services that "Vueltabajo "hotel offer?

Yes No

If you know about these services mention them:

2. Which of the services have you consumed?

3. Which were the better services offered by the hotel?

4. What did motivate you to make use of this hotel's services?

(Mark three answers like maximum)

Relationship quality-price.

Prestige of the hotel.

Friends' recommendations.

Quality of the service.

To ignore other hotels with similar services.

The place where the hotel is situated (near places of your interest).

Previous visits / Knowing of the installation.

Tranquility / Security.

Business / job.

Other reasons: 
5. What procedure does the hotel use to know your satisfaction starting from the services that it gives?

6. Are frequency and rigor applied them the instruments conceived in the procedure to measure the levels of satisfaction with what?

Frequency Rigor

Daily _ Severe

Monthly__ Affable

None

7. Do you believe that these instruments allow to expose in a complete way your opinion?

Yes No

8. Are the parameters that are exposed in this instruments enough? Yes No

9. According to your opinion would you add or what would you subtract to this procedure to make it more effective when the information will be pick up?

10. What importance does it have for you in the execution of this procedure?

11. What are the problems or the inconvenients for this procedure?

12. How would you qualify the quality of this procedure?

Right

Regular

Wrong

13. Do you know another ways that the hotel uses for knowing its criterion about the quality of the given services?

For your cooperation thank you 